\title{
Temporal Evolution of Multi-Carrier Complexes in Single GaN/AlGaN Quantum Dots
}

\author{
K. Surowiecka*, A. Wysmołek, R. Stȩpniewski, R. Bożek, \\ K. Pakula And J.M. Baranowski \\ Institute of Experimental Physics, Warsaw University \\ Hoża 69, 00-681 Warsaw, Poland
}

\begin{abstract}
Microphotoluminescence of low-density GaN/ $\mathrm{Al}_{x} \mathrm{Ga}_{1-x} \mathrm{~N}$ quantum dots grown by metal-organic vapor phase epitaxy using in situ etching of AlGaN is presented. The narrow lines in the microphotoluminescence spectra due to the single quantum dots are observed. Both energy and intensity of these lines show temporal fluctuations. Statistical analysis based on the correlation matrix allowed us to identify objects, which are affected by photo-induced electric field fluctuations. Relations between emission lines participating in the spectrum are discussed.
\end{abstract}

PACS numbers: 78.55.Cr, 78.67.Hc

\section{Introduction}

Standard method of quantum dot (QD) fabrication relays on the StranskiKrastanov growth mode [1]. This technique provides usually high-density assembles of quantum dots, which makes experimental studies of individual quantum dots very difficult. Recent advance in the growth of a new type of quantum dots allowed detecting emission from single GaN/AlGaN QDs using standard microphotoluminescence $(\mu \mathrm{PL})$ technique, without any artificial mesa or mask structures $[2,3]$. In spite of the technological success the knowledge about the formation process and electronic structure of such dots is still very limited. Moreover, the number of the observed luminescence lines is still too high to assign them to given quantum objects.

*corresponding author; e-mail: ksurow@fuw.edu.pl 
In order to shed more light into the subject temporal evolution of the emission from single $\mathrm{GaN} / \mathrm{Al}_{x} \mathrm{Ga}_{1-x}$ N QDs was studied.

\section{Samples and experiment}

The low-density GaN/ $\mathrm{Al}_{x} \mathrm{Ga}_{1-x} \mathrm{~N}$ QDs were grown by metal-organic vapor phase epitaxy (MOVPE) using modification of the proposed earlier method [4]. The primary $\mathrm{Al}_{x} \mathrm{Ga}_{1-x} \mathrm{~N}(x=0.10)$ layer was grown in a standard way starting with a low temperature $\left(700^{\circ} \mathrm{C}\right)$ AlN buffer layer, followed by a $2 \mu \mathrm{m}$ layer grown at $1100^{\circ} \mathrm{C}$ under 200 mbar pressure. In the next step in situ etching of the AlGaN surface in the presence of $\mathrm{SiH}_{4}$ at high temperature was performed. Subsequent GaN deposition led to the formation of crystallites (see Fig. 1a), which were covered by AlGaN layer to form quantum dots. The details of the growth procedure are described elsewhere [3].
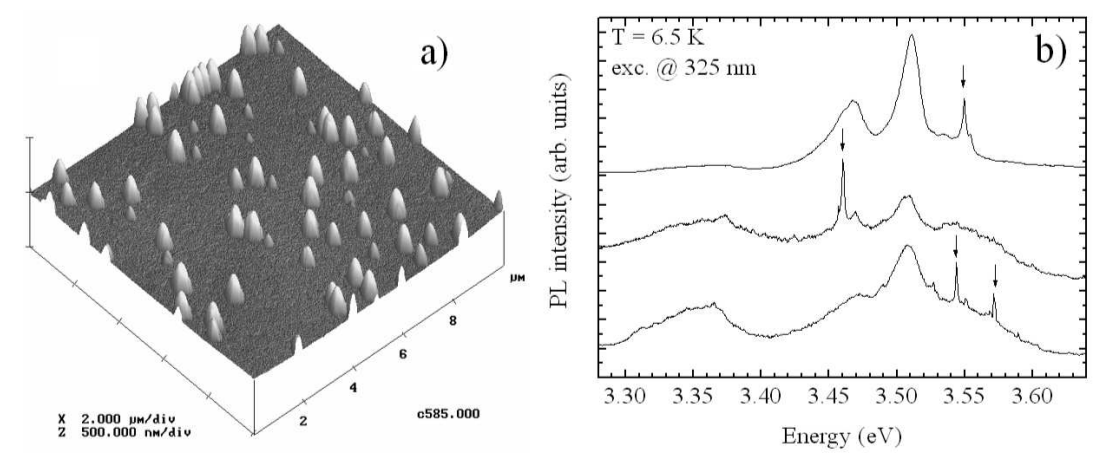

Fig. 1. (a) Separated GaN (AlGaN) crystallites on the AlGaN layer. (b) The $\mu \mathrm{PL}$ spectra of single quantum dots collected for different positions on the sample.

The low temperature $\mu \mathrm{PL}$ was done using $\mathrm{He}-\mathrm{Cd}$ laser operating at $325 \mathrm{~nm}$ line. The measurements were performed using a continuous flow cryostat in temperature range $4.2-60 \mathrm{~K}$. The laser spot was focused on the samples using reflecting microscope objective with the magnification of 25 . Resulting excitation spot size is estimated to be of about few $\mu \mathrm{m}$ in diameter.

\section{Results and discussion}

Typical $\mu \mathrm{PL}$ spectra measured for different positions on the sample are presented in Fig. 1b. They show sharp lines originating from single quantum dots. It is clearly seen that the observed intensity pattern depends on the position of the sample (see Fig. 1b).

When repeating the measurements, it was noticed that PL spectrum changes with time. In order to study details of this effect subsequent spectra were collected. 


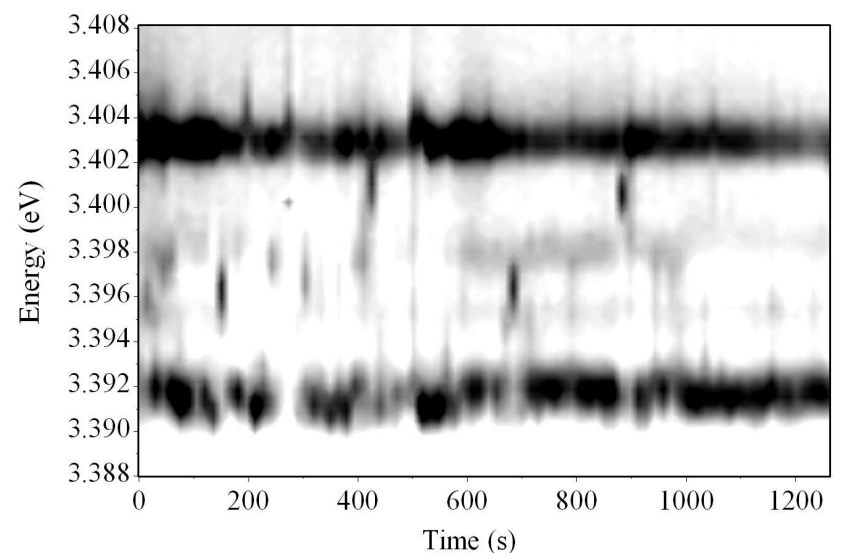

Fig. 2. Spectrally resolved intensity map representing the time evolution of the emission from QDs measured at 6.5 K. Single spectrum was collected for $15 \mathrm{~s}$.

Representative results of such experiment are presented in Fig. 2. It is clearly seen that random fluctuations in the intensity and energy of the emission lines in the spectrum take place. Moreover, some correlations between different transitions are present. For instance, the appearance of the line at $3.396 \mathrm{eV}$ is unambiguously associated with the increase in the intensity of the line at $3.391 \mathrm{eV}$. In order to get more insight in the observed phenomena correlation matrix was calculated for the measured spectra, using the formula

$$
r(m, n)=\frac{\sum_{i}\left(I_{m}^{i}-\left\langle I_{m}\right\rangle\right)\left(I_{n}^{i}-\left\langle I_{n}\right\rangle\right)}{\sqrt{\sum_{i}\left(I_{m}^{i}-\left\langle I_{m}\right\rangle\right)^{2}} \sqrt{\sum_{i}\left(I_{n}^{i}-\left\langle I_{n}\right\rangle\right)^{2}}},
$$

where $I_{m}^{i}$ and $I_{n}^{i}$ symbolize intensities of the pixel $m$ and $n$ in the spectrum number $i$. Two examples of correlation maps obtained from the experimental data are presented in Fig. 3. The correlation coefficient is represented by a gradual change between white (positive correlation) and black (negative correlation) colors. In Fig. 3a two bright spots located symmetrically at the diagonal close to the position corresponding to the pixel 162 are observed. They are accompanied by two symmetrical, off-diagonal black regions. As seen in the original spectra (bottom part in Fig. 3a) such a pattern results from the energy fluctuation of the emission line.

Slightly different pattern is presented in Fig. 3b. However, in this case it corresponds to the negative correlation of the intensity measured at two neighboring lines (around pixels 212 and 220). The observation of negative correlation of these lines is confirmed by direct comparison of the time dependence of the luminescence intensity (Fig. 4). It is seen that the increase in the intensity of the first line is associated with the intensity decrease in the second one. Such a behavior can be expected for the different excitonic complexes originating in the same quantum dot. The energy separation between these lines, being of $2 \mathrm{meV}$ is 
a)

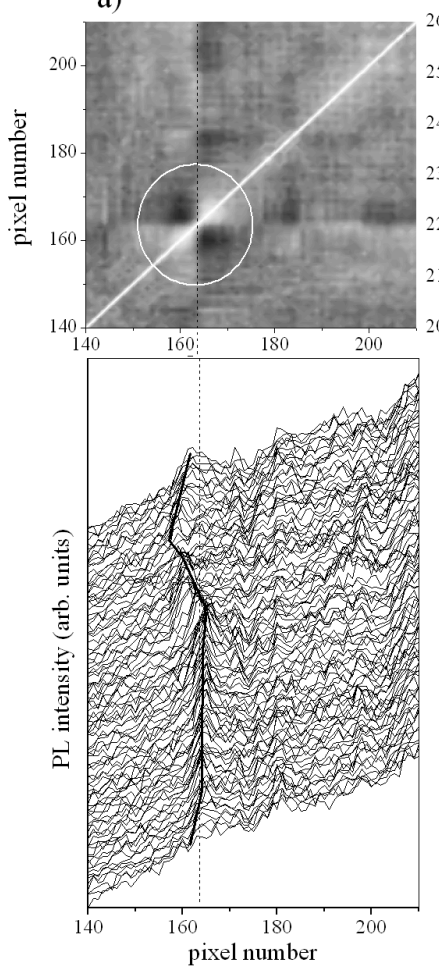

b)
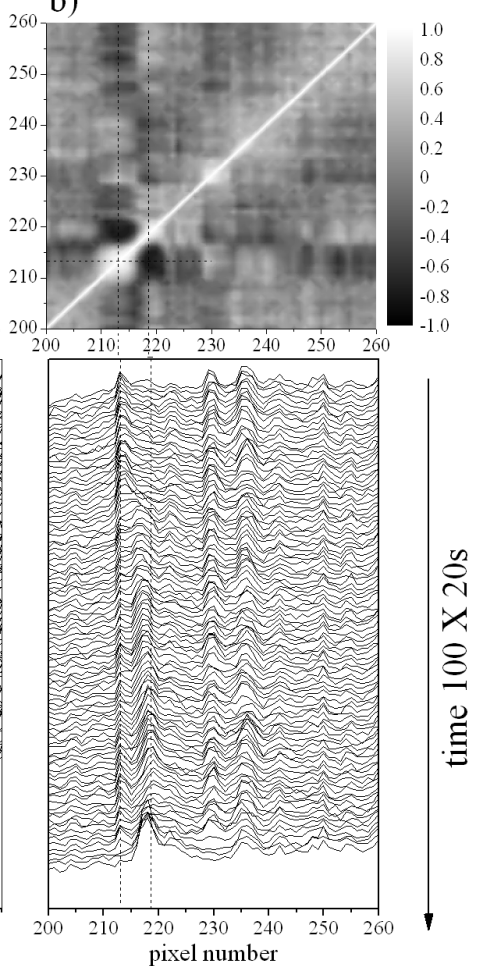

Fig. 3. The manifestation of emission energy (a) and intensity (b) variations in the correlation map. For comparison temporal evolution of the selected part of the $\mu \mathrm{PL}$ spectra is shown (lower part).

in a good agreement with expected difference between exciton and charged exciton binding energy.

As seen from these examples, the correlation matrix calculations can be used as an effective tool allowing identification of the correspondence between different parts of the PL spectrum. First of all, it allows recognizing lines with fluctuating intensity. Such lines manifest their presence through the bright spots at the diagonal of the correlation map. On the other hand, the lines, for which the intensity changes have statistical character only (noise), do not introduce any visible contribution to the correlation map (see Fig. 3b). Bright and dark spots, visible at off-diagonal regions signalize possible relations that occur between different lines identified in the spectrum. However, to interpret properly these relations one has to identify the physical origin of the observed fluctuations.

Random intensity and/or energy fluctuations have been already observed for other QD systems [5-8]. The observed changes in the spectrum can be induced by several sources. The simplest are connected with vibrations of the microscope objective and instabilities of the exciting light intensity. These effects lead ob- 


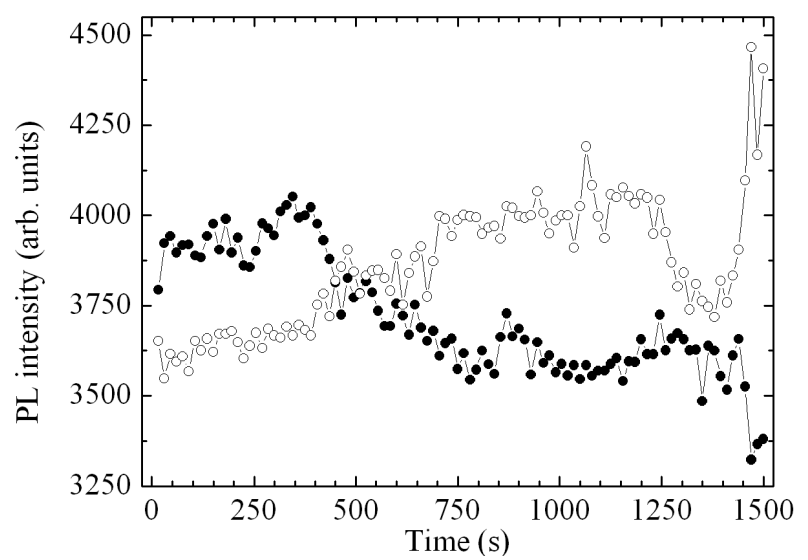

Fig. 4. The photoluminescence intensities of the two negatively (shown in Fig. 3b) correlated lines measured as a function of time.

viously to the correlated changes of the emission intensity corresponding to the recombination within a single quantum dot as well as in the quantum dot ensemble. More indirect source of the intensity and/or emission energy fluctuations can result from the photo-induced redistribution of charges in the dot vicinity, accumulated on structural and point defects. This can lead to the modification of the QD's confining potential that results in the change of the emission energy. On the other hand, the redistribution of carriers could easily influence the QD charge state. Instead of neutral exciton recombination charged excitons and more complicated complexes could be formed. Thus, the negatively correlated doublets, shown in Figs. 3 and 4, can indeed be related to the recombination of neutral and charged excitons. However, in order to confirm such hypothesis additional experiments are required. Thanks to the applied statistical method, the selection of the single objects for further studies was greatly facilitated.

\section{Conclusions}

$\mu \mathrm{PL}$ spectra of GaN/GaAlN structures grown using on AlGaN using high temperature in situ etching show sharp emission lines corresponding to individual QDs.

The observed temporal behavior of the $\mu \mathrm{PL}$ is discussed in terms of charge fluctuation in a medium surrounding QDs. The correlation analysis allowed us to identify transitions originating at the same quantum dot and provided information about the formation of different excitonic complexes in the QDs. The presented method can be useful as a selection tool allowing extracting emission attached to the single quantum dots which contributes in the rich emission spectrum. 


\section{Acknowledgments}

This work was partially supported by the State Committee for Scientific Research grant No. 3T08A 00127.

\section{References}

[1] K. Tachibana, T. Someya, Y. Arakawa, Appl. Phys. Lett. 74, 383 (1999).

[2] B. Chwalisz, A. Wysmołek, R. Bożek, R. Stȩpniewski, K. Pakuła, P. Kossacki, A. Golnik, J.M. Baranowski, Acta Phys. Pol. A 105, 517 (2004).

[3] K. Pakuła, R. Bożek, K. Surowiecka, R. Stẹpniewski, A. Wysmołek, J.M. Baranowski, to be published.

[4] K. Pakuła, R. Bożek, J.M. Baranowski, J. Jasiński, Z. Liliental-Weber, J. Cryst. Growth 267, 1 (2004).

[5] B. Pattron, P. Langbein, U. Woggon, Phys. Rev. B 68, 125316 (2003).

[6] V. Türck, S. Rodt, O. Stier, R. Heitz, R. Engelhardt, U.W. Pohl, D. Bimberg, R. Steingrüber, Phys. Rev. B 61, 9944 (2000).

[7] N. Panev, M.-E. Pistol, J. Persson, W. Seifert, L. Samuelson, Phys. Rev. B 70, 073309 (2004).

[8] K. Kowalik, O. Krebs, A. Kudelski, A. Golnik, A. Lemaître, P. Senellart, G. Karczewski, J. Kossut, J. Gaj, P. Voisin, Acta Phys. Pol. A 106, 177 (2004). 